\title{
Migraine aura without headache: transient ischaemic attack or not?
}

\author{
Martin Dennis, Charles Warlow
}

\begin{abstract}
Patients with "migraine aura without headache" can be separated from those with transient ischaemic attacks (TIA) on the basis of the onset of symptoms, which is generally gradual and which spread or intensify over minutes or hours, and from the typical migrainous nature of their visual symptoms. Fifty cases were prospectively identified and these were compared with 50 age matched patients with a TIA. Surprisingly, there were no significant differences in the prevalence of vascular risk factors and diseases in the two groups although those with TIAs tended to have more. Only one patient with "migraine aura without headache" suffered a subsequent vascular event (a myocardial infarction). Three patients with TIAs had strokes and two others died from vascular disease. Although the results are not conclusive, they do suggest that patients with the clinical characteristics of "migraine aura without headache" have a low risk of subsequent vascular events, lower than those with TIAs, despite perhaps having similar prevalence of vascular risk factors. Although their risk factors should be treated, patients with "migraine aura without headache" should be reassured and not subjected to inappropriate and potentially hazardous investigations and treatment.
\end{abstract}

Focal cerebral and visual symptoms commonly precede or accompany headache in classical migraine. Patients who have classical migraine with neurological symptoms often have similar neurological symptoms at other times but without headache. In such cases, few would disagree that the isolated neurological symptoms are "migrainous" and they have been called "migraine sine hemicrania,", "migraine accompagnee,", "migraine associee", 2 "metastases of migraine,"3 "migraine accompaniments", 4 or "acephalgic migraine". 6

A more difficult clinical problem arises when patients present with transient focal neurological or visual symptoms and yet have never experienced these symptoms in the context of a classical migraine attack, and who have no associated headache. Such patients may present relatively late in life and have symptoms of vascular disease (for example, angina) and risk factors (for example, hypertension) increasing both the doctors' and patients' anxiety that the symptoms, neurological or visual, may be due to cerebral or ocular ischaemia - that is, transient ischaemic attacks (TIAs).

Several authors have described patients in whom the onset and nature of the symptoms suggests that they have a variant of migraine but in whom there is no headache. Perhaps, the most notable reports are those of Fisher ${ }^{45}$ who described "late onset migraine accompaniments" and Whitty ${ }^{8}$ who described "migraine without headache". Fisher has provided many detailed case histories that have helped to delineate the clinical syndrome. $\mathrm{He}$ also stated that "the condition can be justifiably regarded as benign" 5 but without providing any detailed follow up on the patients. $\mathrm{He}$ appears to have relied upon the fact that many of the patients had had attacks for many years when he first saw them and the few he followed up suffered no serious sequelae. Although his conclusion may be correct, the evidence is unreliable since patients who are dead or severely disabled are unlikely to present a neurologist with a history of relatively minor events, nor will they be able to attend follow up clinics. We therefore present the results of a follow up study of a series of patients, clinically similar to those described by Fisher, with what we have called "migraine aura without headache", to describe more precisely the natural history of this condition. We have compared this series of patients with an age and sex matched group of definite TIA patients to determine whether the prevalence of vascular diseases, risk factors, and prognosis is different in the two groups. This is what one would expect if Fisher is correct, that "migraine aura without headache" is a benign condition from the point of view of not just the lack of headache, but prognosis for serious vascular events.

\section{Patients and Methods}

Between 1977-86 patients who were referred to a neurology clinic with transient neurological symptoms were prospectively classified as having definite TIAs or variants of migraine, among other diagnoses, by one of us (CW). Detailed histories including the presence or absence of vascular risk factors were recorded for all patients. Four hundred and sixty nine patients were diagnosed as having definite TIAs ${ }^{9}$ using a standard definition (see below). Another 75 patients were classified as having a variant of migraine although at that time we had no set diagnostic criteria for these cases. At the end of this period the records of the 75 patients were reviewed without knowledge of 
their outcome and diagnostic criteria were applied so that a homogeneous group of patients with "migraine aura without headache" could be described.

Our diagnostic criteria for "migraine aura without headache" were that: either firstly, the focal neurological symptoms should come on gradually and spread or intensify over a period of minutes, not seconds, or secondly, that the patient describes positive visual symptoms characteristic of classical migraine even if they come on abruptly-that is, fortification spectra, flashing lights, dazzles; and thirdly, that headache should be either totally absent or if present at all, should be described by the patient as mild, and that the patient does not take analgesics or change their activity due to the headache; and also fourthly, the patient should not have had similar symptoms previously in the context of either classical migraine or in association with a more severe headache.

By applying these diagnostic criteria to the 75 patients with migraine variants, 25 were excluded, leaving a group of 50 with "migraine aura without headache". The main reason for exclusion was that the patients described moderate or severe headache in association with the presenting or previous episodes.

The 50 patients were stratified by age (into five year age bands) and by sex. We then randomly selected age and sex matched patients from the TIA group which had been similarly stratified. Unfortunately, there were insufficient young female TIA patients in our cohort group to provide age and sex matched controls for each of the patients with "migraine aura without headache". In four cases, therefore, we randomly selected an age matched male patient since age is a more important determinant of the risk of vascular events than sex.

For the purposes of this study, TIA is defined as "an acute loss of focal cerebral or monocular function with symptoms lasting less than 24 hours and which after adequate investigation is presumed to be due to embolic or thrombotic vascular disease". ${ }^{10}$ The patients with "migraine aura without headache" were followed up by telephone in March and April 1987 and asked questions about symptoms which suggested a diagnosis of stroke or myocardial infarction. Where patients described symptoms suggesting a serious vascular problem, their general practitioner and hospital notes were reviewed to confirm the diagnosis. In the majority of other patients the hospital notes were reviewed. The TIA patients were prospectively followed up until the end of 1986 at a specialist clinic, by their general practitioner, or by letter. If patients either died or described symptoms suggesting a serious vascular event, their hospital notes were reviewed to confirm the diagnosis. The prognosis in the two groups was compared using a logrank analysis of Kaplan Meier survival curves. No patient in either group was lost to follow up.

\section{Results}

The general characteristics of the patients with "migraine aura without headache" and TIAs are summarised in table 1 . Forty nine of the fifty patients $(98 \%)$ with "migraine aura without headache" described visual symptoms which were binocular in 35 patients $(71 \%)$, monocular in five $(10 \%)$ and uncertain in nine (18\%). Most patients (35/49; 71\%) described positive visual features such as flashing lights, fortification spectra or haloes. Fifteen patients $(30 \%)$ had other symptoms; most commonly sensory $(13 / 50 ; 26 \%)$, aphasia $(8 / 50 ; 16 \%)$, or dysarthria $(3 / 50 ; 6 \%)$, and clumsiness or weakness of a limb or limbs $(5 / 50 ; 10 \%)$. Some patients described dysgraphia $(2 / 50 ; 4 \%)$ in association with dysphasia, associated vertigo, and feelings of remoteness. One patient lost consciousness during an attack. Patients often gave histories going back over several months or years and described multiple attacks during that period. Only $11(22 \%)$ patients with "migraine aura without headache" and 15 $(30 \%)$ with TIAs were referred with their first attack. The mean number of attacks and length of history was longer in the migraine group than in those with TIAs. Most commonly patients $(23 / 50 ; 46 \%)$ described their symptoms as coming on or intensifying over a period of between six and 30 minutes. A few patients with visual symptoms $(10 / 49 ; 20 \%)$ described a more sudden onset (less than 1 minute), 14 (28\%) had symptoms developing over a period of one to five minutes and a few patients $(3 / 50$; $6 \%$ ) described symptoms coming on over more than 30 minutes. Episodes lasted less than 15 minutes in five patients $(10 \%), 15$ to 60 minutes in $37(74 \%)$ and more than one hour in $8(16 \%)$. Ten patients $(20 \%)$ experienced slight headache, one patient vomited and two others felt slight nausea in association with their visual or neurological symptoms. Two patients with "migraine aura without head-

Table 1 General characteristics and prevalence of vascular diseases and risk factors in patients with "migraine aura without headache", TIA controls, and all the TLA patients referred during the study period

\begin{tabular}{|c|c|c|c|}
\hline & $\begin{array}{l}\text { Migraine } \\
\text { aura without } \\
\text { headache } \\
n=50\end{array}$ & $\begin{array}{l}T I A \\
\text { controls } \\
n=50\end{array}$ & $\begin{array}{l}\text { All TLAs' } \\
n=469\end{array}$ \\
\hline $\begin{array}{l}\text { Mean age (SD) } \\
\text { No of Men (\%) } \\
\text { Hypertension (\%) } \\
\text { Ischaemic heart disease (\%) } \\
\text { Peripheral Vascular disease (\%) } \\
\text { Diabetes (\%) } \\
\text { Current smokers (\%) }\end{array}$ & $\begin{array}{l}48 \cdot 7(13 \cdot 3) \\
30(60) \\
10(20) \\
4(8) \\
3(6) \\
1(2) \\
15(30)\end{array}$ & $\begin{array}{l}49 \cdot 0(13 \cdot 7) \\
34(68) \\
13(26) \\
4(8) \\
5(10) \\
2(4) \\
20(40)\end{array}$ & $\begin{array}{l}62 \cdot 1(12) \\
317(68) \\
198(42) \\
99(21) \\
76(16) \\
25(5) \\
221(47)\end{array}$ \\
\hline
\end{tabular}


Table 2 A comparison of prognosis in patients with "migraine aura without headache" and TLAs with p value based on a logrank analysis

\begin{tabular}{|c|c|c|c|c|c|}
\hline Group & $n$ & $\begin{array}{l}\text { Observed } \\
\text { strokes }\end{array}$ & $\begin{array}{l}\text { Expected } \\
\text { strokes }\end{array}$ & $\begin{array}{l}\text { Observed/ } \\
\text { Expected }\end{array}$ & \\
\hline TIA & 50 & 3 & 1.5 & $2 \cdot 0$ & \multirow{3}{*}{$1 p=0.04$} \\
\hline $\begin{array}{l}\text { Migraine aura without } \\
\text { headache }\end{array}$ & 50 & 0 & 1.5 & 0.0 & \\
\hline Group & $n$ & $\begin{array}{l}\text { Observed vasc deaths/ } \\
\text { strokes/MI }\end{array}$ & $\begin{array}{l}\text { Expected vasc deaths/ } \\
\text { strokes/MI }\end{array}$ & $\begin{array}{l}\text { Observed/ } \\
\text { Expected }\end{array}$ & \\
\hline TIA & 50 & 5 & 3.0 & $1 \cdot 7$ & \multirow{2}{*}{$1 p=0.05$} \\
\hline $\begin{array}{l}\text { Migraine aura without } \\
\text { headache }\end{array}$ & 50 & 1 & 3.0 & $0 \cdot 3$ & \\
\hline
\end{tabular}

ache" gave a history of classical migraine years before but their aura had been quite different from the symptoms described during the presenting episode. Ten other patients gave histories of past headache without other features of migraine.

The prevalence of vascular diseases and risk factors in the patients with "migraine aura without headache" and control TIAs was not significantly different although the TIA patients did have a higher prevalence of all vascular disease and risk factors other than ischaemic heart disease (see table 1). The control TIAs were younger than the whole group of TIA patients referred to the clinic over the study period and almost certainly as a result had a lower prevalence of vascular diseases and risk factors. Seventeen of the 50 patients with TIAs had amaurosis fugax, eight of these were less than 50 years old, a group known to have few risk factors and a good prognosis. Forty seven (94\%) of the TIA patients had an onset of symptoms over only a few seconds. In three patients (6\%) symptoms came on between 1 and five minutes. These three were obviously less classical in their presentation but had been diagnosed as definiteTIAs when they first presented. For the purposes of the analysis in this paper they were not subsequently reclassified. This might have resulted in smaller differences between the TIA and "migraine aura without headache" groups.

During a mean follow up period of 4.5 years ( 1.1 to 9.4 years), one patient $(2 \%)$ with "migraine aura without headache" had a nonfatal myocardial infarction, none died and no patient suffered a stroke (table 2). One patient required coronary artery surgery for angina pectoris and another had arterial surgery for intermittent claudication. During a mean follow up period of 4.9 years $(0.1$ to 9.7 years $)$ six TIA patients died (12\%), three from malignancy, two from definite cardiac disease and one suddenly, presumably from a vascular event. Three $(6 \%)$ TIA patients had a stroke, one of these died subsequently of heart disease, but none suffered a non-fatal myocardial infarction. The prognosis of the two groups in respect of their risk of stroke, myocardial infarction, and vascular death is shown in table 2. During a mean follow up period of $4 \cdot 1$ years, $58(12 \%)$ of all 469 TIA patients died from a vascular cause, $63(13 \%)$ had a stroke and 16 (3\%) had a non-fatal myocardial infarction.

\section{Discussion}

We have described a group of patients who presented with transient focal neurological or visual symptoms and a benign prognosis. Because of the lack of severe headache the diagnosis of TIA was considered but because of the gradual onset or nature of the symptoms described, these patients were classified as having "migraine aura without headache". Since there are no investigations to confirm or refute the diagnosis of "migraine" or of TIA, one has to rely on indirect evidence to decide whether it is reasonable to classify patients in this way. For example, if patients with "migraine aura without headache" had a much lower prevalence of vascular diseases and risk factors than patients with TIAs and also had a lower risk of developing subsequent vascular complications, it would be clinically useful to separate these groups of patients and one might deduce that the underlying pathogenesis was different. It would, of course, be more difficult to say that these patients definitely had migraine, one could only say that whatever the mechanism of the symptoms, the clinical syndrome has a good prognosis and sounds like migraine.

In this study the patients with "migraine aura without headache" had a similar prevalence of vascular diseases and risk factors as those with TIAs. Obviously with only small numbers of patients in each group, only large differences could reliably be demonstrated and type two errors are likely. One explanation of this similarity, if it is indeed true, might be that the symptoms of "migraine aura without headache" may be related to the presence of vascular pathology as in TIAs. It has been suggested that migraine attacks can be triggered by embolic events ${ }^{11}$ but our study cannot further this debate. An alternative, and in our view, a more likely explanation might be that general practitioners and others seeing patients with transient neurological symptoms with some of the features of migraine might refer patients with vascular disease and risk factors to a specialist neurology clinic leading to a referral bias. The control TIA patients in this study had less associated vascular disease and fewer risk factors than unselected TIA patients (see table 1) almost certainly because they were relatively young. More encouraging was the result of our follow up study which despite small numbers suggested the patients with "migraine aura without headache" had a very 
low risk of serious vascular events. This risk appeared to be lower than that in the control TIA patients, although the differences in prognosis did not reach conventional levels of statistical significance; partly because of small numbers but also because we selected younger TIA patients to be controls and by chance included a high proportion (34\%) of patients with amaurosis fugax who have a better prognosis than patients with transient cerebral ischaemic attacks. ${ }^{9}$ This meant that the control TIA patients had a much lower risk of stroke and other vascular events compared with the whole group of TIAs and it became more difficult therefore to show a statistically significant difference between them and the "migraine aura without headache" group. The better prognosis observed in the patients with "migraine aura without headache" could in part be explained by a selection bias; this group may have contained a greater proportion of "survivors" than the TIA group because they presented having had more attacks and with a greater interval since their first ever attack.

We have shown that patients with "migraine aura without headache", according to our criteria, even in the presence of vascular disease or risk factors, have a good prognosis and that it is therefore worth distinguishing them from TIAs. Obviously their vascular risk factors should be treated on merit but potentially hazardous investigations and treatments should be avoided. Generally, patients with "migraine aura without headache" should be reassured that they have a type of migraine, fortunately without headache, which has a very good prognosis, and that they have not had a mild stroke.

1 Bruyn GW. Migraine equivalents. In: Clifford Rose F, ed. Handbook of clinical neurology Vol 4 (48): Headache. Amsterdam: Elsevier, 1986:155-71.

2 Priorry P. Memoire sur une des affections designees sous le nom migraine ou hemicranie. $\mathcal{Y}$ Univ Hebd Med Chir Prat nom migraine

3 Tissot $M$. Le traite de la catalepsie, de l'extase de l'anesthesie, de la migraine, et des maladies du cerveau. Lausanne grasset CY 1788:90-1374.

4 Fisher CM. Late life migraine accompaniments as a cause of unexplained transient ischemic attacks. Can $\mathcal{F}$ Neurol $S c i$ 1980;7:9-17.

5 Fisher CM. Late life migraine accompaniments-further experience. Stroke 1986;17:1033-42.

6 O'Connor PS, Tredici TJ. Acephalgic migraine. Fifteen years experience. Ophthalmology 1981;88:999-1003.

7 Fisher CM. Cerebral ischemia, less familiar types. Clin Neurosurg 1971;18:267-336.

8 Whitty CWM. Migraine without headache. Lancet 1967;ii:283-5.

9 Hankey G, Slattery J, Warlow C. The prognosis of hospital referred transient ischaemic attacks. $\mathcal{f}$ Neurol Neurosurg referred transient ischaemic
Psychiatry 1991;54:793-802.

10 Warlow CP, Morris PJ. Introduction. In: Warlow CP, Morris Warlow CP, Morris PJ. Introduction. In: Warlow CP, Morris
PJ, eds. Transient ischaemic attacks. New York: Dekker, 1982:vii-xi.

11 Peatfield RC. Can transient ischaemic attacks and classical migraine always be distinguished? Headache 1987; 27:240-3. 